\title{
Performance improvement of Arctic tracked vehicles
}

\author{
Roman Dobretsov ${ }^{1, *}$, Gennadii Porshnev ${ }^{1}$, and Darya Uvakina $^{1}$ \\ ${ }^{1}$ Peter the Great St. Petersburg Polytechnic University, Polytechnicheskaya 29, St. Petersburg, \\ 195251, Russian Federation
}

\begin{abstract}
The technical tenders aimed at improving of track-type vehicles performances are considered and substantiated, and that vehicles are the irreplaceable basis of the Arctic transport network. The questions of the improving of reliability and Energy Performance of crawler due to the application of caterpillars with modernized tracks that have a lower mass and provide less resistance to movement of the vehicle are considered. The variants of transmission modernization with the purpose of improving the quality of turn control due to the use of a split-transmission diagram with a steering based on an electric motor and other solutions are proposed. An economically expedient approach to the introduction of a hybrid power plant of a parallel-serial type based on the developed kinematic scheme of a electromechanical split-transmission is proposed. It is shown the main technical proposals can be realize based on domestic units.
\end{abstract}

\section{Introduction}

The sustainable development of Arctic region is impossible without the decision of a transport problem [1-7]. The one aspect of that is to ensure the uninterrupted operation of tracked vehicles having high mobility and sufficient load-carrying capacity in order to move passengers and cargo in the Arctic, or to carry out the armament and protection sufficient for this potential military operations theater.

Amphibious tracked vehicles by one- and two-sectional design most satisfy of the characteristic conditions of operation (such as: snow and ice cover; extremely low temperatures in winter; the presence of extensive areas of soil with low bearing capacity in summer, watered areas, marshes of various types and water barriers, etc.). In Russia, the most famous were the transporters of the DT series of various load-carrying capacity and single-sectional machines on that chassis. Overview of the DT series and technical characteristics of the transporters can be found on the manufacturer's website [8]. The DT series transporters were designed initially for military purposes, but were subsequently adapted for use primarily in the geological exploration and oil and gas industry.

Due to the modern reality for Russia it is necessary to reinforce the Arctic peaceful development by the presence of a military presence. The DT series transporters remain

${ }^{*}$ Corresponding author: dr-idpo@yandex.ru 
successful for locating armament, ensuring delivery of landing and military cargoes. However, these tracked vehicles have some drawbacks, which make difficult to military use them in the future.

The rubber-cord caterpillar, as compared to the linked metal caterpillar, has a smaller ballistic protection and is vulnerable to damage by debris because of a mine explosion. Belt caterpillars are difficult to replace in the field conditions (especially at low temperatures and a snowstorm). The caterpillar, compared with plan pin joint caterpillars, requires more pre-tensioning force, which leads to increase loads on other mover elements. The use of a linked caterpillar with aplan pin joint, subject to a reduction in the mass per unit length and the preservation of high cross-country ability in the snow, can increase the mobility, improve maintainability, and positively affect the economy.

The two-sectional chassis turns due to the relative rotation of the sections on the plane. The coupling (adhesion) unit failure entails loss of controllability of the vehicle and makes the rear section driven, while for overcoming difficult off-roads conditions it is very useful that both transporter sections can generate a propulsive effort. Loads on the coupler are large. The hydraulic drive of sections steering mechanism on the ground with a large resistance to rotation is overloaded, which ensures the impossibility of turning with small radii. The design of the steering practically excludes, for example, a turn with a radius equal to half the width of the vehicle track and a zero-radius turn. For an armed vehicle, turns with small radii are important, the vehicle must have high agility. Accordingly, the caterpillar characteristics can be increased (without significant alteration of the serial sample) due to the use of the steering of a different design, as well as the development of a electromechanical split-transmission designed to operate a hybrid power plant of a parallelseries type.

Studies in the field of improving the caterpillar design aimed at increasing its energy efficiency, mobility, longevity, recoverability, etc., have been conducted for several decades at the Department of Engines, Automobiles and Tracked Vehiclesof SPbPU [9]. Due to achievements of modern technologies, some concepts in the field of improving the caterpillar, proposed by the staff and professors of the department, can be implemented.The work objective is to increase the track vehicles mobility and reduce the energy costs of the caterpillar due to a complex of modernization measures applicable to mass-produced machines.

The aim of the work is to increase the efficiency of tracked vehicle for Arctic region.

\section{Methods and materials}

Vehicle and tracked vehicle caterpillars were the subject of the research.

The methods of theoretical mechanics, terrain mechanics, theory of tracked vehicles, mathematical analysis, probability theory and decision theory, engineering experiment were used in the work.

\section{Results}

The using in the mover of the two-linked arctic off-roads vehicle of linked tracks is irrational mainly due to two factors. A wide metal linked caterpillar with a traditional design has a large mass; for linked tracks, the track instability effect under the support rollers is observed, which is accompanied by an increase in resistance to movement.

Currently a design method and structure of nonmetallic wideners for linked caterpillars are developed [10]. Its elasticity avoids damage and reduces the caterpillar cocking when overcoming artificial obstacles, stone placers, hummocks and standing floes. The wideners shape increases the contact surface area of the caterpillar as it dives into the snow, by 
analogy with the operation principle of a cylinder-conic wheel, described, for example, in the article [11]. A build-up link design (see $[12,13]$ ) has been proposed for reducing the truck mass, the width of which is not more than twice the width of the tire of the rollers The development of this principle could be the introduction of the track, developed according to the approaches of bionic design. It should be expected that in the latter case the truck will be too expensive to manufacture. Another alternative is a steel belt caterpillar (see, e.g., patent [14]). The high cost and difficulty of replacing when operation are its main disadvantages. In this way, a narrow caterpillar with lightweight tracks and non-metallic wideners is preferable.

In Fig. 1 links with a sequential plan pin joint of a traditional construction are shows, and Fig. 2 has tracks with a parallel plan pin joint, made by patent [12]. In the upgraded link, the tension force is acted between the lugs through the power elements such as sidewalls and amplifiers. A link rail is made smooth (a version of the design provides for a partial overlap of the gap between adjacent tracks) and is formed by the surface of the nonmetallic insert. Grousers made at the edge of the link. The links are interconnected with steel pins and buckles. The crests preventing a track from the link rail are made on buckles.

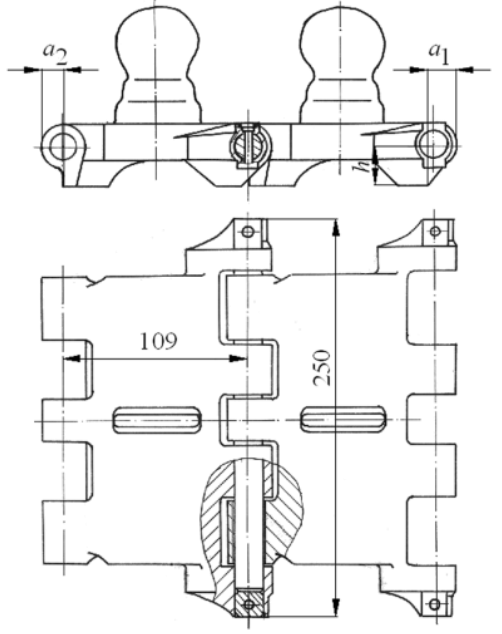

Fig. 1. Series caterpillar tracks of a tracked vehicle model: the maximum arm of a overturn force $a_{1}=a_{2}=10 \mathrm{~mm}$; the distance from the hinge axis to the grouser edge $h=24 \mathrm{~mm}$

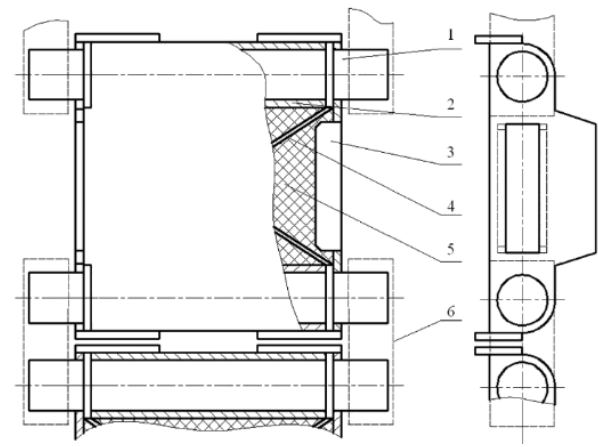

Fig. 2. The diagram of a caterpillar track made by patent [13]: 1 - pins, 2 - lugs, 3 - walls, 4 - insert, 5 - non-metallic insert, 6 - buckles

The listed design features of the upgraded link allow to reduce the arm of a normal force acting when aroadwheel rolls from track to track and causes the overturning of tracks.

The math model of track overturning for caterpillar with a parallel typeis described in [15]. If we designate $\boldsymbol{Y}$ as a set of factors that determine the calculated value of the overturning force (truck geometrical dimensions, in particular, the location of the front edge of the grouser relative to the front edge of the track; tire stiffness, hinge stiffness, weight and dimensions of tracks and roadwheels; design outline, etc.); $\boldsymbol{X}_{R}$ as a coordinate vector of the axis of the roll relative to the front edge of the grouser, then $M_{(i+l)}\left(\boldsymbol{Y}, \boldsymbol{X}_{R}\right)$ is a overturn torque for adjacent tracks $i$ and $i+1$ in general. Denote by $t$ as a step of a link, and write the condition of the absence of a track turning relative to the line passing through the front edges of grousers:

$$
M_{i(i+l)}\left(\boldsymbol{Y}, \boldsymbol{X}_{R}\right) \leq 0, \forall \boldsymbol{X}_{R} \in[-t / 2, t / 2]
$$


The amplitude of the angular oscillations of the "unstable" tracks is small (within a few degrees) and is accompanied by insignificant movements. However, these movements may be accompanied by a positive displacement of the entire vehicle.In experiments on concrete, where it is more convenient to observe such effects, it turned out that losses may exceed $20 \mathrm{~kW}$ for a single-section vehicle with a mass of 40-45 tons at a speed about $10 \mathrm{~m} /$ s. In the case of a two-sectional caterpillar, a kinematic conflict arises: one of the sections begins to push (or pull forward) the other because of the positive displacement of the tracks.

The vehicle movement is also accompanied by other significant energy consumption (for example, dynamic oscillations of the caterpillar outline branches), but for some vehicles due to the suppression of the tracks oscillations, we can expect a reduction in power losses by $8-10 \%$ (see $[15,16])$. The influence of effects, associated with the instability of tracks under the roadwheel, on the chassis energy balance are analyzed in [17] and others. The tracks instability effect is observedclearly with a specific thrust of $0.10 \ldots 0.12$ and is accompanied by a positive displacement of the vehicle. The process of slipping a caterpillarincreases with an increase in traction force (for example, towing a trailer). The ideal rolling mode of a mover is a transitional stage.

The increase in longitudinal force leads not only to an increase in slippage, but also to the effect of redistributing loads along the length of the supporting surface: the front road wheels and the caterpillar part under them are unloaded and are not participated in the transmission of normal and tangential reactions. The mover model in this mode is described in the articles $[18,19$, etc.]. Effects associated with trucks instability may develop on the underloaded part of the caterpillar.

Single-linked tracked vehicles intended for the carriage of passengers and cargo in the Arctic region often have a transmission that is similar to the transmission of a conveyorswamp vehicle in structure (Fig. 3). The turning is controlled by changing the thrust forces on the driving rolls (this function is performed by the steering).

The transmission is similar for the front link of the two-sectional vehicle, but there is no steering and power take-off is provided for the active rear link drive. The front and rear links are coupled with a hitch, providing the transmission of torque to the transmission of the rear active link and steering control due to the relative reversal of the sections in the plane. The hitch allows angular movement of links in the vertical plane.

Given in Fig. 3 the solution is quite typical for various purposes tracked tractors and forestry vehicles based on their chassis [20,21], transporters and other vehicles [22]. In this way, for all the vehicles listed above, there are common drawbacks: the lack of a unique connection between the position of the control device and the turning radius; turning radius changes in spurts; the minimum turning radius is equal to half the track width for most structures. 


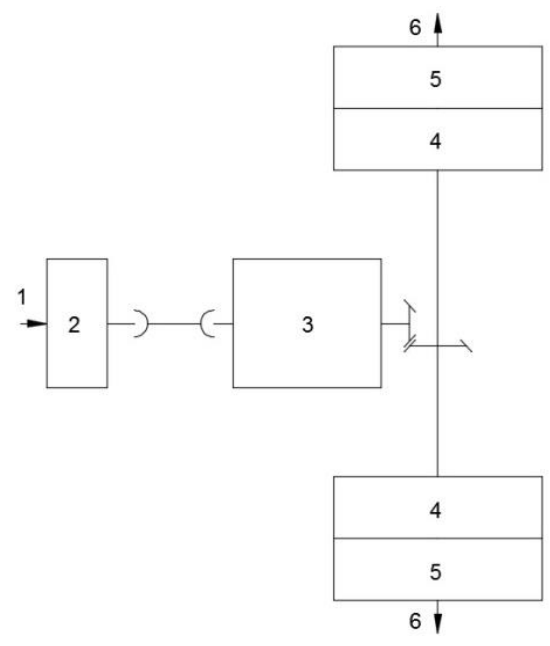

Fig. 3. Simplified block diagram of a tracked vehicle transmission with a central gearbox and onboard steering mechanisms: 1 -power supply from the internal combustion engine (ICE); 2 -coupling mechanism; 3 - gearbox; 4 - steering mechanism; 5 -final drive; 6 - power transfer to the driving wheel

For two-linked vehicles, problems associated with overloading the hydraulic drive of the section steering mechanism and the large value of the minimum turning radius are typical.

To provide an unambiguous connection between the position of the control device and the radius in the whole range of operating conditions is possible only due to the use of closed turning control systems. This problem was considered, in particular, in $[23,24]$ and others. The principle of applying pulse-width modulation of pressure in the system of hydraulic drive of clutch disk controls of the steering mechanism in order to control the slipping of a disk pack has the greatest prospects. This principle is convenient for application, in particular, on planetary steering mechanisms with hydraulic drive. Such a mechanism is the cheapest and can functionally replace the common mechanically driven side friction clutch. Some prospects are opened by the use of power distribution mechanisms as steering mechanisms (ZF Vector Drive [25], AYC [26], domestic developments [27]).

However, only a split-transmission can provide the best quality steering control. In the transmission, the main power flow passes from the engine through the gearbox to the summing planetary gear sets. Preferably, the change of the steering mechanism gear ratio occurs smoothly. Power flows are combined on summing planetary sets and sent to the sides driving wheels.

Traditional diagrams of split transmissions are presented in the book [21]. Hydrostatic gear [28,29], friction gear [30] and an electric motor can be applied in a parallel power flow.

A simplified diagram of the upgraded transmission is presented in Fig. 4.

For applying a traction motor, it is necessary to significantly increase the power of the generator (from the practice of designing electromechanical power transmissions, the term "traction electric generator" (TEG) is borrowed) and to provide an electrical energy storage device.

Differential 5 (see. Fig. 4) in a parallel power flow can expand the number of operating modes of the transmission. In fact, the resulting version of the transmission corresponds to the concept of a hybrid power plant of parallel-serial type.The group of related solutions for 
military vehicles, differing from off-road vehicles by the arrangement, was considered earlier in article [31].

The materials of conference about the using of hybrid power plants on vehicles (see the collection in which the article [31] was published) show that at present the domestic industry can manufacture compact and lightweight electrical motors and energy storage devices operating in the required temperature range.

Transmission option in fig. 4,a is a diagram in fig. 4,b where a reversible electric machine (REM) is provided. This decision will lead to the complication of the kinematic diagram and control algorithms; it will be necessary to install a larger capacity drive. However, this solution is more reasonable for applying on machines with a compact engine compartment.

Shown in fig. 4,a transmission diagram allows the following operation modes.

Rectilinear motion using ICE. ICE should work in the maximum efficiency mode for greater rationality. Aelectrical motor is connected with a parallel branch of the transmission through the gearbox (position 11 in Fig. 4). The reduction gear ratio is selected from the condition the required range of turning radii. A symmetrical cylindrical differential (position 5 in Fig. 4) is installed in the parallel branch, with two control elements: a brake $T_{R}$ and a locking clutch $C_{R}$. In the case of straight-line movement of the machine, the electrical motor is disabled, the parallel branch is not used: the brake $T_{R}$ and the clutch $C_{R}$ are on. Rectilinear motion is steady. The electrical energy produced by TEG is accumulated in the storage device and can also be used to power other users.

Turn with ICE and electrical motor. The clutch $C_{R}$ is disengaged when turning. The brake $T_{R}$ is on. The electrical motor turns on (depending on the turning direction, the rotation direction of the shaft traction motor is selected). The running side speed increases, and of the lagging side decreases. The power supply of the traction motor is carried out from the energy storage device and the TEG, therefore the total power at the driving wheels can be briefly higher than the power of the ICE.

Turn with 0-radius using only electrical motor. The electrical motor is activated in the same way as in the previous mode, but the engine is not used (neutral in the gearbox) or disabled. The main branch of the transmission is stopped. The maximum power of the traction motor is selected based on the ability to provide this mode.

Rectilinear motion with the use of internal combustion engines and the traction motor (forced mode). The clutch $C_{R}$ is included in the parallel branch. Both transmission branches are used.

Rectilinear motion using only electrical motor (standby mode). If the main branch fails (the main branch shafts are stopped), the vehicle can move due to the electrical motor (storage energy is used). The clutch $C_{R}$ is activated in a parallel branch, the stopping brakes of the sides are used to control the direction of movement, since the movement will be carried out only at low speeds.

Turn only with the ICE (standby mode). In case of failure of the parallel branch of the transmission, the machine can be operated using the stopping brakes of the sides $T_{0}$. The clutch $C_{R}$ and the brake $T_{R}$ are turned on in parallel branch. The vehicle can move at a low speed to the repair.

A variant of the diagram according to fig. 4,b allows to implement the same modes of operation. The REM, when used in the electrical motor mode, is connected to a parallel flow by a clutch $C_{2}$. At another time, it works in the generator mode (connected by a control element in the gearbox housing). 


\section{EECE-2018}

a)
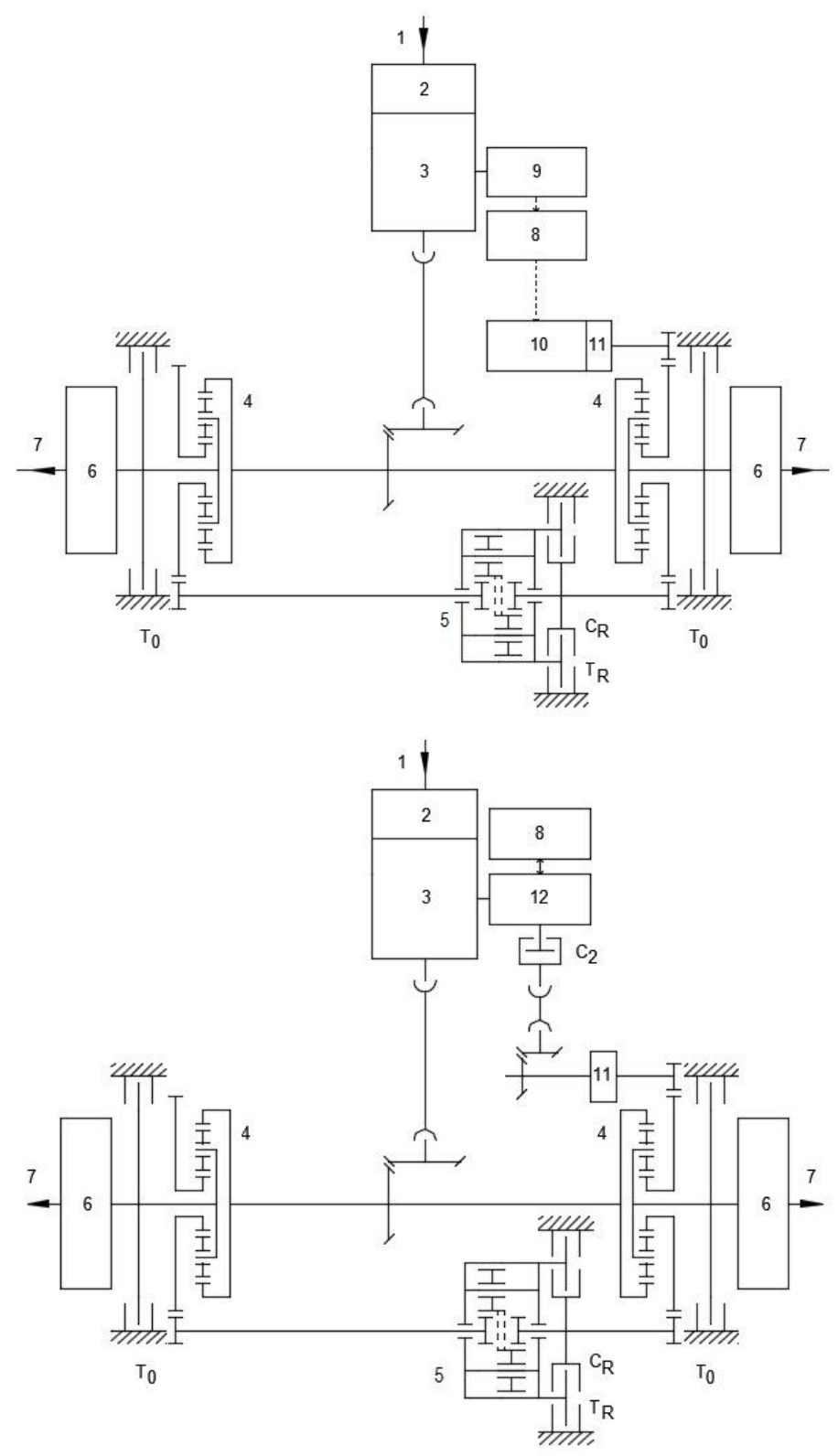

Fig. 4. Kinematic diagram of split-transmission: a -with traditional electric machines; $b$-with reversible electric machine; 1 -power supply from engine, 2 - coupling mechanism; 3 - gearbox; 4 summing planetary gear set, 5 -differential with controls $C_{R}$ and $T_{R}, 6$-final drive, 7 -power transfer to the driving wheel, 8 - storage device, 9 - traction electric generator, 10 - electrical motor, 11 parallel flow reverse gear, 12 - reversible electric machine, $\mathrm{T}_{0}$ - stopping brake, $\mathrm{C}_{2}-$ connection clutch of REM

To calculate the power of the electrical motor, we will consider a turn around with 0 radius [31] in difficult ground conditions (the turn on the snow proceeds with less energy consumptions, but the vehicle must also function in summer off-road conditions).

The required power of the electric motor in this mode can be calculated as: 


$$
\left.N\right|_{\rho=0}=M \omega / \eta_{3 \Pi}
$$

Here $\rho=R / B$ - relative turning radius $(R-$ turning radius); $M=\mu G L / 4-$ torque of resistance to turning $\left(G-\right.$ vehicle weight, $L$ - bearing length); $\omega=\left(V_{2}-V_{1}\right) / B$ - turning angular velocity $\left(V_{2} \mathrm{u} V_{1}-\right.$ linear running and lagging velocities); $\eta_{3 \Pi}-$ gearing efficiency.

Dependence $\mu(\rho)$ is described by an empirical formula:

$$
\mu=\mu_{\max } /(0,925+0,15 \rho)
$$

The value of the coefficient of maximum resistance to rotation was determined empirically when turning the tracked vehicle around the stopped side [29]:

$$
\mu_{\max }=\left.\mu\right|_{\rho=0,5}
$$

For estimated calculation, we use this dependence, assuming the hyperbolic nature of the change $\mu(\rho), \rho \in[0 ; 0,5]$ and carrying out an extrapolation.

For a single-section caterpillar with a mass of 11,6 ton with values of $L=2,3 \mathrm{~m}$ and $B=$ $1,7 \mathrm{~m}$, with $\eta_{3 \Pi}=0,85$ and an angular velocity of rotation $\omega=0,2 \mathrm{rad} / \mathrm{s}$ on summer offroad conditional, we obtain the power of the electrical motor about $26,6 \mathrm{~kW}$. This value is about $1 / 3$ of the engine power installed on a modern the third traction class tractor. It can be expected that the mass of the electrical motor of such power, made by the brushless diagram, fits into the range of $40-70 \mathrm{~kg}$.

In this way, when using the diagram in fig. 4 , a at the same time as improving the steering control quality, the integration of the hybrid power plant into the chassis of the production vehicle is obtained. The rational applying of a hybrid power plant will improve the efficiency of economy, at the same time giving the opportunity to significantly increase the thrust force.

The diagram in fig. $4, \mathrm{~b}$ at the present stage seems less promising: the advantages achieved by replacing two electric machines with one, are offset by the complexity of the transmission. Such a scheme can be adapted for military or military vehicles [28,29].

\section{Conclusions}

1. The applying of metal linked caterpillars with upgraded links can increase the reliability and and maintainability of the vehicle without special equipment, increase the durability of the propulsion unit, and reduce the energy consumption for moving the vehicle.

2. The use of a hybrid parallel-type power plant, made according to the proposed deagram, should increase the mobility and efficiency of the vehicle and improve its manageability.

3. Currently, there are technologies in the Russian Federation that make it possible to implement proposals for upgrading tracked vehicles based on components of domestic production.

4. Improving the technical characteristics of tracked vehicles (first of all - reliability, recoverability, efficiency and mobility) will strengthen the position of Russian Federation and ensure security in the Arctic region.

\section{References}

1. I.E. Frolov, Stud. Russ. Econ. Dev. 26, 561 (2015).

2. V.N. Leksin and B.N. Porfiryev, Stud. Russ. Econ. Dev. 27, 418 (2016).

3. V.N. Borisov and O. V Pochukaeva, Stud. Russ. Econ. Dev. 27, 159 (2016). 
4. N.I. Komkov, V.S. Selin, V.A. Tsukerman, and E.S. Goryachevskaya, Stud. Russ. Econ. Dev. 27, 180 (2016).

5. E. V Rytova, A. V Kozlov, S.S. Gutman, and I.M. Zaychenko, J. Adv. Res. Law Econ. 7, 1828 (2016).

6. S.S. Gutman, I.M. Zaychenko, and E. V Rytova, in (International Business Information Management Association, IBIMA, 1439-1449 (2017).

7. A. V Grinëv, J. Slav. Mil. Stud. 31, 272 (2018).

8. http://www.bolotohod.ru/ru/cat1/dt_info/

9. Yu.V. Galishev, R.Yu. Dobretsov, G.P.Porshnev, S.I. Hoodorozhkov, St. Petersburg State Polytechnic University Journal of Engineering Science and Technology, 1(190), 26-32 (2014).

10. A.A. Anikin, Science and education: electronic scientific and technical publication, 8 (2010).

11. E.V.Avotin, R.Yu.Dobretsov, S.I.Matrossov, St. Petersburg State Polytechnic University Journal of Engineering Science and Technology, 3(178), 230-237 (2013).

12. R.Yu. Dobretsov, A.G. Semenov, Caterpillar of the vehicle undercarriage. Pat. 2385815 Russian Federation, IPC B62D 55/20. (2010)

13. R.Yu. Dobretsov, A.G. Semenov, Russian forests and its economy, 1-2 (42-43), 34-35 (2012).

14. R.Yu. Dobretsov, A.G. Semenov, A.V. Smirnov, Elastic Vehicle Caterpillar. Pat. RU 2446975 C1 Russian Federation, IPC B62D 55/54 (2012)

15. R.Yu. Dobretsov, Trac. andagricul. veh., 6, 25-31, (2009).

16. R.Yu. Dobretsov, I.V. Grigoriev,V.A. Ivanov, Sys. Meth. Techn, 2(30), 114-119 (2016).

17. R.Yu. Dobretsov, St. Petersburg State Polytechnic University Journal of Engineering Science and Technology, 3, 98-103 (2011)

18. Yu.V. Galyshev, R.Yu. Dobretsov, St. Petersburg State Polytechnic University Journal of Engineering Science and Technology, 3, 272-278 (2013).

19. R.Yu. Dobretsov, I.V. Grigoriev, Russia's Forests: Politics, Industry, Science, Ed. V.M. Gegyo., 124-127 (2016).

20. N.B. Veselov, Cross-country transport and technological vehicles. Structures. Design and calculation. (2010)

21. V.I. Patyakinand [et al.] Technology and equipment of forest industry. Textbook (2009)

22. V.F. Platonov, A.F. Belousov, N.G. Oleynikov, G.I. Kartsev; ed. by V.F. Platonov, Tracked transporters (1978)

23. Yu.V. Galyshev [et al.], St. Petersburg State Polytechnic University Journal of Engineering Science and Technology, №3,2, 201-208 (2014)

24. R. Dobretsov, I. Grigorev, A. Gazizov, Vestnik Bash. St. Agr. Univ., 3 (43), 97-106 (2017)

25. http://www.irs.kit.edu/download/131213 GC TorqueVectoring_ZF_Handout.pdf

26. Y. Ushiroda, K. Sawase, N. Takahashi, K. Suzuki, K. Manabe., Development of Super AYC,Tech. rev., 15, 73-76 (2003)

27. R.A. Didikov [et al.] International Scientific Conference Energy Management of Municipal Transportation Facilities and Transport EMMFT 692, 192-200 (2018) 
28. N.A. Nosov, V.D. Galyshev, Yu.P. Volkov [et al.], Calculation and design of tracked vehicles: A textbook for universities (1972)

29. V.B. Shelomov, The theory of movement of multi-operated tracked and wheeled vehicles. Traction calculation of curvilinear motion: a manual for universities in the specialty "Automobile and tractor" (2013)

30. R.Yu. Dobretsov, Inventors in the innovation process in Russia, 121-124 (2014)

31. R.Yu. Dobretsov [et al.], Collection of articles of the scientific-practical conference "Development and applying of electric transmissions for weapons and military equipment (JSC "Mobile Vehicle Engineering Institute"), 87-100 (2016) 